\title{
A national epidemiological study investigating risk factors for police interrogation and false confession among juveniles and young persons
}

\author{
Gisli H. Gudjonsson ${ }^{1,2}$ • Jon Fridrik Sigurdsson ${ }^{2,3}$ • Inga Dora Sigfusdottir ${ }^{2}$. \\ Bryndis Bjork Asgeirsdottir ${ }^{2} \cdot$ Rafael A. González $^{4,5} \cdot$ Susan Young $^{2,4,6,7}$
}

Received: 9 July 2015 / Accepted: 13 October 2015/Published online: 4 November 2015

(C) The Author(s) 2015. This article is published with open access at Springerlink.com

\begin{abstract}
Purpose The principal aims of this study are to identify risk factors associated with police arrest and false confessions and to investigate whether the severity of the ADHD condition/symptoms increases the risk.

Methods 22,226 young persons in Iceland anonymously completed self-report questionnaires screening for conduct disorder and ADHD. In addition, they stated whether they had a diagnosis of ADHD and had received ADHD medication, and their history of offending, police interrogation and false confession. Participants were stratified into two age groups, 14-16 and 17-24 years.

Results The older group was significantly more likely to have been interrogated by the police but the younger group were much more vulnerable to false confession during interrogation. Males were more likely to be at risk for both than females. The severity of the ADHD condition increased the risk of both interrogation and false confession. Negative binomial regressions showed that age,
\end{abstract}

Susan Young

susan.young1@imperial.ac.uk

King's College London, London, UK

2 Reykjavik University, Reykjavík, Iceland

3 University of Iceland and Landspitali-The National University Hospital of Iceland, Reykjavík, Iceland

4 Imperial College London, London, UK

5 University of Puerto Rico Graduate School of Public Health, San Juan, USA

6 Broadmoor Hospital, WLMHT, London, UK

7 Faculty of Medicine, Centre for Mental Health, Imperial College London, 7th Floor Commonwealth Building, Du Cane Road, London W12 0NN, UK gender, conduct disorder, offending, and ADHD symptoms were all significant predictors of both interrogations and number of false confessions. Conduct disorder was the single best predictor of police interrogation, but the findings were more mixed regarding false confessions. Young people presenting with a combination of severe ADHD and comorbid conduct disorder had the worst outcome for both interrogation and false confessions.

Conclusions The findings endorse the need for support of persons with ADHD to be put in place to ensure fair due process and to prevent miscarriages of justice.

Keywords Epidemiology · Interrogation · False confessions · ADHD · Conduct disorder · Offending

\section{Introduction}

It is important to identify risk factors associated with police interrogation and false confessions in order that appropriate safeguards may be applied. Age is an important predictor of outcome. A review of the literature revealed that juveniles are more vulnerable to giving a false confession during interrogation than adults [1-5]. The extent of offending behaviour (OB) is also predictive of false confessions both among juveniles in community samples [6,7] and adult prisoners [8]. Males are more likely to be interrogated than females [6], but uncertainties remain regarding the gender risk for giving a false confession due to the small number of female false confession participants in previous studies. The large sample in the current study should give a definitive answer regarding the role of both age and gender in interrogations and false confessions.

Given the high rates of ADHD reported in the prison population of $26 \%$ for adults and $30 \%$ for youths [9], it is 
often assumed that ADHD is a salient risk factor for offending. The risk, however, may be more attributed to conduct disorder (CD; or antisocial personality disorder in an older group) due to the irresponsible life style and disregard for the consequences of behaviour associated with these disorders [10]. CD and ADHD are associated conditions [11-13] with a common genetic influence [14]. Young and Gudjonsson [15] have demonstrated the importance of antisocial personality traits in people clinically diagnosed with ADHD, which makes them susceptible to social maladjustment and delinquency. Lynam [16] suggests that children with a combination of ADHD and $\mathrm{CD}$ are at greatest risk of becoming persistent offenders. Recent research has suggested that the relationship between ADHD and offending is largely mediated by conduct disorder (CD), substance misuse and association with delinquent peers [17]. It is likely that those mediating factors bring them to the attention of police rather than their ADHD symptoms per se, whereas their ADHD may leave them additionally vulnerable to falsely confessing to a crime during interrogation.

Previous work has not attempted to tease out the relationship between predictors of arrests and false confessions. Frequency of arrests has been predicted by both an ADHD diagnosis and childhood CD symptoms, the latter being the more powerful predictor than the former [18]. Loeber, Burke, Lahey, Winters, \& Zera [19] found that CD is a major risk factor in terms of later criminal behaviour and police arrests. Satterfield et al. [20] conducted a 30-year follow-up of 179 clinically referred and treated hyperactive boys and 75 controls, assessed in childhood when aged 6-12 years. A high rate of adult arrests (44.1\%) was found in contrast to controls $(14.7 \%)$, with the ADHD group being 4.57 times more likely to be arrested than controls, 4.68 more likely to be convicted and 4.08 times more likely to be incarcerated. The highest rate of arrest (59\%) was between the ages of 18-21 and the rate declined with age. Most of the sample (78\%) had childhood behavioural symptoms consistent with CD. Langley et al. [21] in a 5-year follow-up of 126 children diagnosed with ADHD and treated in childhood (mean age 9.4 years), found that $61 \%$ reported at least one police contact at follow-up (mean age 14.5 years). Police contact in the previous 3 months was $27 \%$. At follow-up, $31.5 \%$ were diagnosed with $\mathrm{CD}$ and $63 \%$ were currently prescribed stimulant medication.

So far as false confessions are concerned, Gudjonsson, Sigurdsson, Bragason, Einarsson, \& Valdimarsdottir [22] found that antisocial personality traits and the extent of general offending were highly predictive of false confessions among college and university students. In a prison sample, Gudjonsson, Sigurdsson, Einarsson, Bragason, \& Newton [23] reported that $41 \%$ of prisoners who were symptomatic for ADHD had a history of false confession in contrast to $18 \%$ of non-ADHD prison controls. ADHD was found to predict false confessions above antisocial personality disorder [24] suggesting that the high rate of false confessions reported among the ADHD group was not significantly mediated by their antisocial personality disorder. An epidemiological study reported a similar finding [11].

What has not been systematically investigated for age, gender, $\mathrm{ADHD}, \mathrm{CD}$ and $\mathrm{OB}$ is whether different factors pose a risk for being arrested for the purpose of interrogation and false confessions. The present study aimed to address this gap in knowledge by conducting a study with definitive power to investigate whether the pattern of risk for juveniles and young persons to be arrested and interrogated by the police for suspected crimes is similar to the risk that they will give a false confession. The literature reviewed suggests that the severity of ADHD symptoms is likely to be an important mediator of outcome, thus the present study included measures of severity.

$\mathrm{CD}$ and $\mathrm{OB}$ are likely to be salient triggers for arrest and interrogation, whereas ADHD symptoms per se are probably less important at the point of arrest. In contrast, once arrested and interrogated ADHD symptoms are likely to become more relevant to how they cope with interrogation in terms of the risk of giving a false confession [24].

The current sample was stratified into two age groups, 14-16 and 17-24 years. This categorisation is based on the UK Police and Criminal Evidence Act 1984 (PACE) Code of Practice where those below the age of 17 are classified as 'juveniles' and require the services of an 'Appropriate Adult' during police interviews. The role of an 'Appropriate Adult' is to provide support and advice to people with intellectual disability and other mental health difficulties and is the main protection for juveniles and 'mentally vulnerable' detainees during interviews by police [25].

Hypothesis 1 is that older youth (17-24) are more likely to be interrogated by police than juveniles (14-16), whereas the younger group is likely to report more instances of false confessions during interrogation. Hypothesis 2 is that males are more likely to be interrogated than females and when interrogated they are more likely to give false confessions. Hypothesis 3 is that $C D$ and $\mathrm{OB}$ are better predictors of interrogation than false confessions, whereas ADHD is a better predictor of false confessions than $\mathrm{CD}$ and $\mathrm{OB}$. Hypothesis 4 is that the severity of the ADHD condition/symptoms increases the risk for police interrogation and false confession. 


\section{Method}

\section{Participants}

The sample was comprised of 22,226 young persons in Iceland; $10,838(48.8 \%)$ were in the final 3 years of mandatory education and 11,388 (51.2\%) were in further education at college. The mean age for the total sample (496 did not give their age) was 16.4 years $(\mathrm{SD}=1.9$; range $14-24$ years). There were 10,778 males (49 \%) and 11,211 females (237 did not give their gender). For the purpose of analysis, the participants were categorised into two age groups, those aged $14-16(n=13,933)$ and $17-24$ $(n=7797)$.

144 schools of mandatory education in Iceland were represented in the study. The current sample included $86 \%$ of all mandatory students in Iceland at the time of data collection which took place in February 2012. In Iceland, $95 \%$ of those who finish mandatory education go into further education in colleges. With regard to the college students, all 40 colleges of further education in Iceland were represented. The current sample included $70.5 \%$ of all students registered in the colleges at the time of the data collection, which took place at the end of 2010 and beginning of 2011. We have no information on those students who did not participate in the Survey. The students who did not complete the survey were primarily those who did not turn up for scheduled class on the day of the Survey.

All the schools and pupils consented to take part in the survey. Approval was provided by the Icelandic Ministry of Education and the survey was conducted in accordance with the Icelandic Science Ethics Committee ethical code of conduct, as well as national law.

\section{Measures}

A detailed survey questionnaire asked about participants' family circumstances, education, mental health problems, offending, police involvement and false confession [11].

The survey measures included the following:

Barkley Current Symptoms Scale (BCS) [26]. This scale corresponds with DSM-IV criteria for ADHD symptoms. Each of the 18 items, nine items relating to inattention and nine items to hyperactivity/impulsivity, is scored on a 4-point rating scale for frequency of symptoms experienced during the previous 6 months. Scores range between 0 and 27 for each of the two subscales (Inattention and Hyperactivity/Impulsivity) and 0-54 for the Total scale. In the current study, a screening diagnosis for ADHD symptoms was obtained if six or more of the inattention or hyperactivity/impulsivity items were endorsed as either 'often' or 'very often' (i.e. 6 out of the 9 items had to be endorsed on either subscale). This is the scoring criterion used in previous research [11, 24, 27].

Questions about ADHD diagnosis and medication. Participants were specifically asked 'Have you been diagnosed with ADHD?' and 'Are you currently taking medication for ADHD?'. Both answers were endorsed as either 'Yes' or 'No'.

Severity of ADHD. Two measures of severity were obtained from (a) categorising the symptomatic group into predominantly inattentive, predominantly hyperactive/impulsive, and combined type; the combined type represents greatest severity (see Tables 1,2); and (b) combining those who are currently self-reporting ADHD symptoms and to be receiving ADHD medication (which implies that the medication may not be fully effective in reducing symptoms below screening diagnostic threshold). These are different but overlapping measures of severity. In the latter case this resulted in a hierarchy of presentation according to severity of symptoms as follows, which takes into consideration both current ADHD symptoms and medication status (see Table 4):

Severity 1 : not on medication and not meeting screening diagnosis on BCS $(N=19,492 ; 91.4 \%)$

Severity 2: not on medication but meeting screening diagnosis on BCS $(N=868 ; 4.1 \%)$

Severity 3 : currently on medication but not meeting screening diagnosis on BCS $(N=791 ; 3.7 \%)$

Severity 4: currently on medication and meeting screening diagnosis on BCS $(N=179 ; 0.8 \%)$

The oregon adolescent depression project conduct disorder screen (OADP-CDS) [28]. This 6-item self-report screen of adolescent conduct behaviours, rated on a 4-point Likert scale, provided a total score ranging between 6 (no endorsement of any behaviour) and 24 (maximum endorsement of each behaviour). The OADPCDS has been shown to have good internal consistency, test-retest reliability, and good screening efficiency for detecting lifetime conduct disorder [28]. A cutoff score of 10 or higher was used as an indicator of the presence of conduct disorder.

Offending behaviour $(O B)$ [29]. This five-item scale measures the extent of self-reported offending. The question asked is: "How often have you done the following?" and five delinquent behaviours are rated (e.g. minor theft, major theft, violence, vandalism and burglary) during the previous 12 months. Answers range from 1 (never) to 7 (18 times or more). We dichotomised the group categorically: a score of 1 (no offending) versus a score of 2 or higher (offending). 
Police interrogation and confessions questionnaire [11]. Participants were asked about their experiences of police interrogation, confessions and false confessions as follows:

'How often have you been interrogated at a police station as a suspect in a criminal offence?' and 'Have you ever confessed during police interrogation to a criminal offence that you did not commit (i.e. you had nothing to do with the offence and are completely innocent)?' Replies were rated on the five-point scale: 'Never', 'Once', 'Twice', '3-5 times', ' 6 or more times'.

Because in the authors' previous experience participants have been reluctant to specify a precise number for these variables, thereby leaving a great deal of missing data, these otherwise count variables were coded in ordered categories.

\section{Procedure}

The participants were approached by teachers in scheduled classes and invited to participate in the survey. The participants were assured that their answers would be anonymous. The questionnaire took up to $80 \mathrm{~min}$ to complete and upon completion the students sealed them in a blank envelope and left it by the exit of the classroom.

\section{Analytical strategy}

Frequencies were reported for all categorical variables, and means with their standard deviations for continuous descriptive variables.

To establish independence in the proportions of the observations of all binary and categorical variables, we used Chi-square $\left(\chi^{2}\right)$ tests. For all these binary association tests, odds ratios (OR) with $95 \%$ confidence intervals were calculated as a measurement of their effect size.

Taking into account the proportion of zero responses, and their overall distribution we treated the response variables number of interrogations and of false confessions as count, and fitted negative binomial regression (NBR) models. A Poisson distribution is appropriate in accounting for observed heterogeneity when using count data but is not when there is overdispersion, as observed in both these response variables. And although there was a high proportion of zero responses on both variables, a zero-inflated model presupposes the existence of two zero processes (i.e. two plausible reasons why there are zero responses) [30]. In our context, NBR was the most appropriate. For each multivariate model of number of interrogations and number of false confessions, the following variables we entered simultaneously: age group ( $<17,17$ or more), gender, CD, $\mathrm{OB}$ and ADHD-symptomatic.
Model beta coefficients were exponentiated, with odds ratios (OR) as indicators of the magnitude of associations in binary outcome models, and incidence rate ratios (IRR) in negative binomial regressions. A significance level of $\alpha<0.05$ was adopted throughout. All analyses were performed using Stata version 13 [31].

\section{Results}

\section{Base rates of interrogation and false confession}

Out of 21,260 participants where data were available, 2987 $(14.0 \%)$ reported having been interrogated at a police station. Of those, 1739 (58.2\%) had been interrogated only once, $586(19.6 \%)$ twice, $408(13.7 \%)$ three to five times, and $254(8.5 \%)$ six or more times. Males were significantly more likely to report having been ever interrogated than females, 19.8 and $8.7 \%$, respectively $\left(\chi^{2}=542.2, d f=1\right.$, $p<0.001$, OR $=2.6,95 \%$ CI 2.4-2.8), as well as those in the older age group (21.3 versus $10.1 \% ; \chi^{2}=369.5$, $d f=1, p<0.001$, OR $=2.2,95 \%$ CI 2.0-2.4).

As far as the reporting of false confessions is concerned, of those 2947 participants interrogated where data on false confessions were available, $434(14.7 \%)$ reported having made a false confession; out of those, 264 (60.8\%) had made a false confession once, $82(18.9 \%)$ made a false confession twice, $40(9.2 \%)$ three to five times, and 48 $(11.1 \%)$ six or more times. Males were significantly more likely to report having made a false confession than females, 16.2 and $11.4 \%$, respectively $\left(\chi^{2}=11.7, \quad d f=1\right.$, $p<0.001 ;$ OR $=1.5,95 \%$ CI 1.2-1.9). Those in the younger age group $(20.0 \%)$ were more likely than those in the older group $(10.3 \%)$ to report having given a false confession $\left(\chi^{2}=50.9, d f=1, p<0.001, \mathrm{OR}=2.2,95 \%\right.$ CI 1.7-2.7).

\section{Base rate for predictors of vulnerability}

Out of 22,226 participants, 1097 (4.9\%) met screening criteria for self-reported ADHD symptoms, of whom 469 $(2.1 \%)$ were predominantly inattentive type, 273 (1.2\%) hyperactive/impulsive type, and 353 (1.6\%) were combined type. $2288(10.8 \%)$ reported having received a diagnosis of $\mathrm{ADHD}$ and 946 (4.5\%) reported to be currently taking medication for ADHD. The great majority $(76.8 \%)$ of those of the comorbid type reported not being currently on ADHD medication. Of the total sample, $3098(14.3 \%)$ met screening criteria for CD and $4207(20 \%)$ reported having committed one or more offences. 
Table 1 Differences in the predictor variables between those interrogated and those not interrogated

\begin{tabular}{lclrl}
\hline & Interrogated $N(\%)$ & Not interrogated $N(\%)$ & $\chi^{2} d f=1$ & OR $(95 \% \mathrm{CI})$ \\
\hline ADHD-symptomatic & $340(11.4)$ & $698(3.8)$ & $316.2^{*}$ & $3.1(2.8-3.7)$ \\
ADHD-inattentive & $136(4.6)$ & $313(1.7)$ & $100.2^{*}$ & $2.7(2.2-3.4)$ \\
ADHD-hyperactive & $71(2.4)$ & $189(1.0)$ & $38.3^{*}$ & $2.3(1.6-3.1)$ \\
ADHD-combined & $133(4.5)$ & $196(1.1)$ & $192.5^{*}$ & $4.3(3.4-5.4)$ \\
Current medication & $290(9.9)$ & $636(3.5)$ & $245.8^{*}$ & $3.0(2.6-3.5)$ \\
History of diagnosis & $697(23.3)$ & $1564(8.6)$ & $566.8^{*}$ & $3.2(2.9-3.5)$ \\
Conduct disorder & $1153(39.4)$ & $1791(9.9)$ & $1818.4^{*}$ & $5.9(5.4-6.4)$ \\
Offending behaviour & $1187(40.8)$ & $2993(16.6)$ & $919.7^{*}$ & $3.4(3.2-3.8)$ \\
\hline
\end{tabular}

$* p<0.001$

\begin{tabular}{lllrl}
\hline & False confession $N(\%)$ & No false confession $N(\%)$ & $\chi^{2} d f=1$ & OR (95 \% CI) \\
\hline ADHD-symptomatic & $95(21.9)$ & $241(9.6)$ & $55.4^{*}$ & $2.6(2.0-3.4)$ \\
ADHD-inattentive & $26(6.0)$ & $109(4.3)$ & 2.3 & $1.4(0.9-2.2)$ \\
ADHD-hyperactive & $20(4.6)$ & $49(2.0)$ & $11.4^{*}$ & $2.4(1.4-4.1)$ \\
ADHD-combined & $49(11.3)$ & $83(3.3)$ & $55.2^{*}$ & $3.7(2.6-5.4)$ \\
Current medication & $99(24.1)$ & $188(7.6)$ & $108.1^{*}$ & $3.9(3.0-5.1)$ \\
History of diagnosis & $167(40.5)$ & $505(20.4)$ & $80.2^{*}$ & $2.7(2.1-3.3)$ \\
Conduct disorder & $246(59.6)$ & $893(36.1)$ & $81.8^{*}$ & $2.6(2.1-3.2)$ \\
Offending behaviour & $239(58.0)$ & $943(38.1)$ & $57.6^{*}$ & $2.2(1.8-2.8)$ \\
\hline
\end{tabular}

$* p<0.001$

Table 2 Differences in the predictor variables between those giving a false confession and those with no history of a false confession

\section{Models for number of interrogations and false confessions}

Table 3 summarises the outcome of the negative binomial regression models for number of interrogations and false confessions. The predictors entered were: Age group (i.e.

Table 3 Summary of negative binomial regressions for interrogations and false confessions

\begin{tabular}{lrrl}
\hline Explanatory variables & $B(\mathrm{SE})$ & $z$ & IRR $(95 \% \mathrm{CI})$ \\
\hline Interrogations $^{\mathrm{a}}$ & & & \\
Age group ( $\geq 17$ years $)$ & $0.64(0.04)$ & 15.4 & $1.9(1.8-2.1)^{* *}$ \\
Gender & $-0.80(0.04)$ & -18.5 & $0.5(0.4-0.5)^{* *}$ \\
Conduct disorder & $1.25(0.05)$ & 25.8 & $3.5(3.2-3.8)^{* *}$ \\
Offending behaviour & $0.79(0.05)$ & 17.4 & $2.2(2.0-2.4)^{* *}$ \\
ADHD-symptomatic & $0.59(0.07)$ & 7.9 & $1.8(1.6-2.1)^{* *}$ \\
False confessions & & & \\
Age group $(\geq 17$ years $)$ & $-0.74(0.12)$ & -6.2 & $0.5(0.4-0.6)^{* *}$ \\
Gender & $-0.38(0.13)$ & -3.0 & $0.7(0.5-0.9)^{*}$ \\
Conduct disorder & $0.71(0.12)$ & 5.8 & $2.0(1.6-2.6)^{* *}$ \\
Offending behaviour & $0.59(0.12)$ & 4.9 & $1.8(1.4-2.3)^{* *}$ \\
ADHD-symptomatic & $0.67(0.16)$ & 4.3 & $2.0(1.4-2.7)^{* *}$ \\
\hline$* p<0.01 ; * * p<0.001$ & & & \\
${ }^{a}$ LR test, $\chi^{2}(1)=1595.1, p<0.001$ & & \\
b LR test, $\chi^{2}(1)=372.8, p<0.001$ & &
\end{tabular}


14-16 versus $17-24)$, gender $($ male $=1$, female $=2)$; $C D$, $\mathrm{OB}$ and ADHD-symptomatic. A forced entry method was used in view of the theoretical relevance of the predictors to interrogation and false confession. As far as interrogation was concerned, the full multivariate model showed that $\mathrm{CD}$ was the single best predictor (IRR $=3.5,95 \% \mathrm{CI}$ 3.2-3.8), followed by offending behaviour (IRR $=2.2$, $95 \%$ CI 2.0-2.4) and ADHD-symptomatic (IRR $=1.8$, $95 \%$ CI 1.6-2.1). Meanwhile, for number of false confessions, similar effect sizes were found for ADHD $(\mathrm{IRR}=2.0,95 \%$ CI $1.4-2.7), \mathrm{CD}(\mathrm{IRR}=2.0,95 \% \mathrm{CI}$ 1.6-2.6) and age group (IRR $=2.0,95 \%$ CI 1.7-2.5; Note: IRR was inverted to reflect "risk" association).

\section{ADHD vulnerability status, police interrogation and false confession}

The ADHD status of the 21,330 participants (where complete data were available-data were missing for 896 participants or $4 \%$ ) was categorised using the hierarchy of presentation according to severity of symptoms. Table 4 shows that both police interrogation and false confession were linearly related to ADHD status with a medium effect size (Cramer's $V=0.15$ and 0.22 for interrogation and false confession, retrospectively). Only $12.4 \%$ of those who were not symptomatic and not on medication reported having been interrogated in contrast to $48.5 \%$ of those who were medicated and symptomatic. With regard to false confession, the respective percentages were 10.8 and $40.2 \%$

To aid interpretation, the proportions of interrogations and false confessions in relation to severity are depicted graphically in Fig. 1. The severity of symptoms related to being on ADHD medication and still symptomatic was more linearly related to false confessions than interrogations. Meanwhile, interrogated participants who were symptomatic and on medication were disproportionately high when contrasted with those who were either symptomatic, or on medication only.

There was a strong relationship (large effect size) between the severity group classification and CD $\left(\chi^{2}=1595.12, p<0.001\right.$, Cramer's $\left.V=0.275\right)$, with CD being most common among the severity group $4(64.8 \%)$ and lowest among severity group $1(11.4 \%)$. There was a significant difference between those who were medicated and symptomatic versus those medicated and non-symptomatic with the former being more likely to have $\mathrm{CD}$ $\left(\chi^{2}=89.22 ; \quad d f=1, \quad\right.$ Cramer's $V=0.307, \quad p<0.001$, $\mathrm{OR}=4.9,95 \%$ CI $3.5-6.9)$.

A similar pattern was found with respect to $\mathrm{OB}$ during the previous 12 months (medium effect size) $\left(\chi^{2}=409.24\right.$, $d f=3$, Cramer's $V=0.140$ ), with the OB being most common among the severity group $4(50.0 \%)$ and lowest among severity group $1(18.4 \%)$. There was a significant difference between those who were medicated and symptomatic versus those medicated and non-symptomatic with the former being more likely to have offended $\left(\chi^{2}=27.4\right.$, $d f=1$, Cramer's $V=0.173, p<0.001, \mathrm{OR}=2.4,95 \%$ CI 1.7-3.4).

\section{Discussion}

The hypotheses related to both age and gender were supported. Participants in the older age group were twice more likely to have been interrogated than the younger group, but the younger participants were twice more likely to give a false confession when interrogated (both medium effect size). These findings add substantially to the literature and reinforce the preliminary findings of Gudjonsson et al.[6] by clearly demonstrating that younger youth (14-16) are considerably more vulnerable to giving false confessions than older youth (17-24). This may be due to the relative immaturity of the younger age group and their difficulties in coping with interrogative pressure [4, 10].

Males were more likely than females to report an interrogation and a false confession. The effect size was larger with regard to interrogations than false confessions (medium versus small effect size). This suggests that gender is good predictor of whom the police bring in for interrogation; this most likely relates to the higher level of $\mathrm{CD}$ and $\mathrm{OB}$. Gender was a less powerful predictor of false confessions than interrogation, but the current study demonstrated that there was some relationship with the

Table 4 Rate of interrogation and false confession across the four 'diagnostic' groups

\begin{tabular}{lcccc}
\hline Four groups & Interrogated $N(\%)$ & Not interrogated $N(\%)$ & False confession $N(\%)$ & No false confession $N(\%)$ \\
\hline Not symptomatic and not medicated & $2380(12.4)$ & $16,854(87.6)$ & $254(10.8)$ & $2105(89.2)$ \\
Symptomatic and not medicated & $249(29.4)$ & $599(70.6)$ & $57(23.2)$ & $189(76.8)$ \\
Not symptomatic and medicated & $207(27.4)$ & $548(72.6)$ & $66(32.2)$ & $139(67.8)$ \\
Symptomatic and medicated & $83(48.5)$ & $88(51.5)$ & $33(40.2)$ & $49(59.8)$ \\
& $\chi^{2}=493.71^{*} ; d f=3$, Cramer's $V=0.15$ & $\chi^{2}=139.39 * ; f=3$, Cramer's $V=0.22$
\end{tabular}

$* p<0.001$ 
Fig. 1 Proportion of false confession and interrogations in relation to symptom and medication status

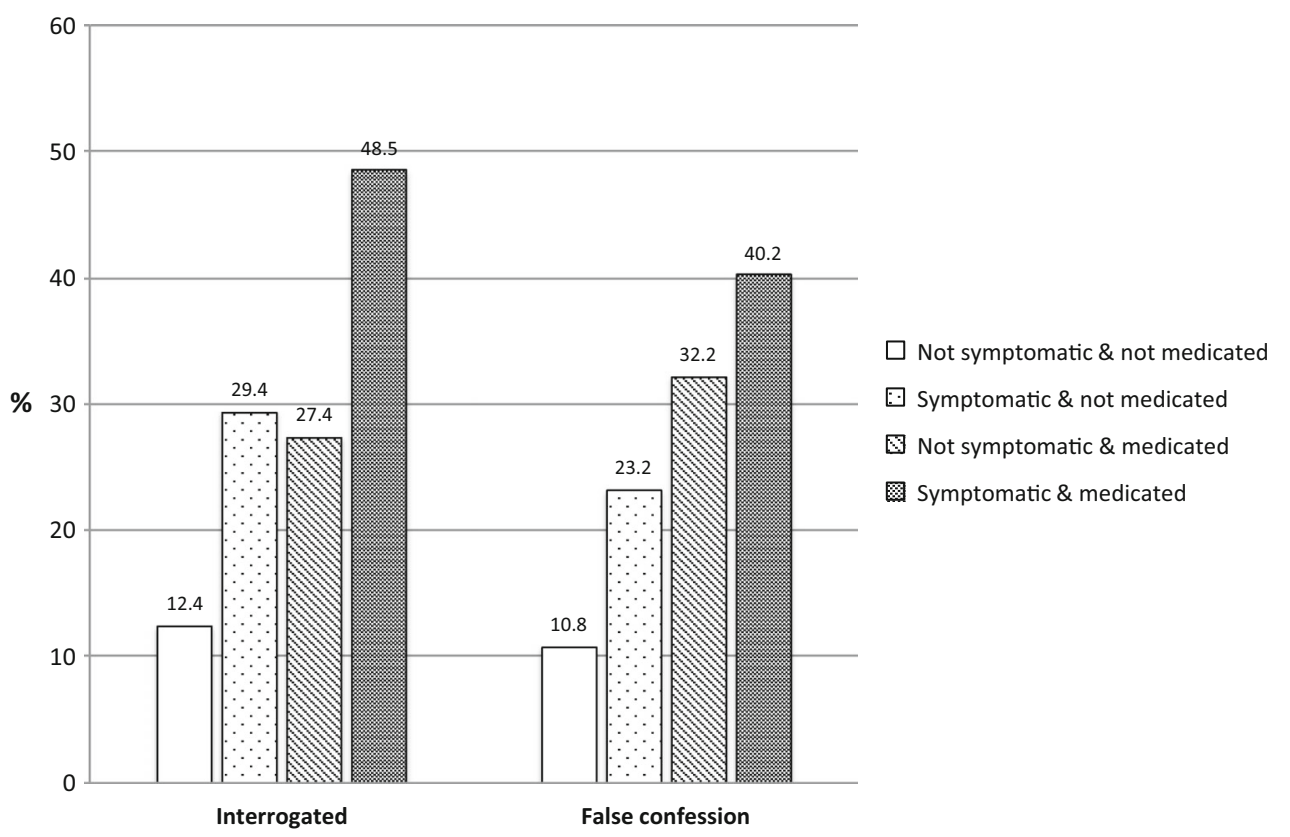

susceptibility to give false confessions. This merits further research, particularly as miscarriages of justice due to unreliable confessions predominantly involve males [32].

As hypothesised, there were differences in the relative contribution of $\mathrm{CD}$ and ADHD in relation to interrogation and false confessions and the severity of ADHD was a further contributory vulnerability factor. A screening diagnosis of CD increased the likelihood of having been interrogated 5.9 times and the likelihood of a false confession 2.6 times. The corresponding ORs for ADHDcombined were 4.3 and 3.7 , respectively. The ORs for ADHD were lower (i.e. 3.1 and 2.6) when including the larger group of participants who met the screening criteria for ADHD-symptomatic. This suggests that the severity of ADHD in terms meeting screening criteria for both inattention and hyperactivity/impulsivity, referred to as the combined type, substantially increases the risk of being brought to a police station for interrogation and giving a false confession. The great majority of these comorbid young persons $(76.8 \%)$ were not being medicated for their condition at the time of the data collection.

NBR regressions showed that age, gender, conduct disorder, offending, and ADHD-symptomatic were all significant predictors of number of interrogations and false confessions. CD made the largest single contribution to the variance in police interrogations and $\mathrm{CD}$ and ADHDsymptomatic to false confessions. The findings suggest that $\mathrm{CD}$ is likely to bring young people to the attention of the police, but when interrogated their ADHD symptoms presents an additional vulnerability to giving a false confessions. This is consistent with research into false confessions among adult prisoners with ADHD [23]. The relative contribution of ADHD to interrogations and false confession in the regression model does not reflect the importance of the severity of the condition, which is well illustrated in Tables 1 and 2, because we included the more commonly used ADHD-symptomatic group rather than the much smaller combined type and those currently on medication.

The contribution to both police interrogation and false confession was also strongly related to our second measure of severity of the ADHD condition with those who were currently symptomatic and on medication (i.e. severity group 4) being most commonly interrogated $(48.5 \%)$ and reporting a history of a false confession $(40.2 \%)$. This vulnerability group, whose medication may be ineffective in reducing their ADHD symptoms, had the highest cocomorbidity with CD $(64.8 \%)$ and $\mathrm{OB}(50.0 \%)$. They resembled the ADHD participants followed up by Satterfield et al. [20] and Langley et al. [21] who responded poorly to stimulant medication and had a high level of CD comorbidity, police contact, and offending. Young people with severe and untreated ADHD are also likely to be at the greatest risk of substance misuse [33] and re-offending [34, 35]. Even though this particular vulnerability group formed a very small part of the overall sample $(<1 \%)$, it is undoubtedly a group that requires the most urgent intervention to prevent future antisocial behaviour and persistent criminal trajectory. The overall effect of the severity of the ADHD condition was stronger for false confessions (Cramer's $V=0.22$ ) than interrogation (Cramer's $V=0.15$ ) and was more linearly related to the four group classification (see Fig. 1), suggesting a more direct relationship with false confessions. 
Taken together, the two different measures of ADHD severity used in the current study, which only overlapped in a minority of cases, provide evidence that the severity of ADHD is an important risk factor in relation to both interrogation and false confession in addition to young persons being merely symptomatic in accordance with diagnostic screening criteria.

The strength of the study is its representative sample of a large national population; there were approximately similar number of participants in both groups and for the two genders. This made it possible to calculate and control for both age and gender differences in relation to interrogation experience and false confessions. In addition, the large sample size enabled the researchers to investigate the relationship of the combination of ADHD symptoms and medication status to both interrogation and false confessions, which has never been done before. The study was limited from its reliance on self-reported data as corroboration of responses was not obtained. Second, the current findings do not provide a complete explanation of factors related to false confession as this is likely to be complex. The false confession or false denial of an offence much depends on the situational context (e.g. the nature and duration of the interrogation, what suits the suspect at a given time) [22], as well as personality and health factors that were not measured in the current study [36].Third, as far as ADHD medication is concerned, it is a limitation that the participants were not asked about medication adherence, which is often a problem with children and adolescents diagnosed with ADHD [34, 37] and likely to be mediated by CD [38]. In Lichtenstein et al.'s [39] landmark study, medication was determined by the prescriptions issued, but there was no data available with regard to adherence. In spite of this, there were 32 and $41 \%$ drop in official offending rate for males and females, respectively, whilst being prescribed ADHD medication. This suggests that ADHD medication may reduce offending and the potential mediators of adherence to medication and comorbid CD should be investigated in future. Multimodal interventions are likely to improve the treatment effect [40] suggesting that medication should be supplemented by programmes developed for this population such as $R \& R 2$ $[41,42]$. Importantly, the findings of the present study raise the possibility that appropriate treatment may reduce the risk of a miscarriage of justice.

In summary, this large national epidemiological study adds to our understanding about the relative importance of $\mathrm{ADHD}, \mathrm{CD}$ and $\mathrm{OB}$ with regard to police interrogation and false confessions. Fourteen percent of the participants reported having been subject to police interrogation and the study highlighted the important contribution of the severity of the ADHD condition and need for effective treatment, together with the need for support to be put in place to ensure fair due process and prevent miscarriages of justice.

Acknowledgments The authors are grateful to the Icelandic Centre for Social Research and Analysis, a research institute focusing on youth research, science studies and science policy, for allowing access to the data and analysis for the purpose of the present paper.

Financial support None of the authors were provided with financial support for the purpose of this study.

Conflict of interest Susan Young has received consultancy fees, speakers fees and/or travel honoraria from Janssen, Eli Lilly, Shire, Novartis and Flynn Pharma, and received research grants from Janssen, Eli Lilly and Shire. Gisli Gudjonsson has received consultancy fees, speaker fees and/or travel honoraria from Eli Lilly, Janssen and Shire. Inga Dora Sigfusdottir is the founder of the Icelandic Centre for Social Research and Analysis. The other others have no conflict of interest.

Ethical standards The authors assert that all procedures contributing to this work comply with the ethical standards of the relevant national and institutional committees on human experimentation and with the Helsinki Declaration of 1975, as revised in 2008.

Open Access This article is distributed under the terms of the Creative Commons Attribution 4.0 International License (http://crea tivecommons.org/licenses/by/4.0/), which permits unrestricted use, distribution, and reproduction in any medium, provided you give appropriate credit to the original author(s) and the source, provide a link to the Creative Commons license, and indicate if changes were made.

\section{References}

1. Drizin SA, Leo RA (2004) The problem of false confessions in the post-DNA world. N C Law Rev 82:891-1007

2. Garratt BL (2011) Convincing the innocent. Harvard University Press, London

3. Gudjonsson GH (2010) The psychology of false confessions: A review of the current evidence. In: Lassiter GD, Meissner CA (eds) Police interrogations and false confessions. American Psychological Association, New York, pp 31-47

4. Kassin SM, Drizin SA, Grisso T, Gudjonsson GH, Leo RA, Redlich AD (2010) Police-induced confessions: risk factors and recommendations. Law Hum Behav 34(1):3-38. doi:10.1007/ s10979-009-9188-6

5. Redlich AD (2007) Double jeopardy in the interrogation room for youths with mental illness. Am Psychol 62(6):609-611. doi:10. 1037/0003-066X62.6.609

6. Gudjonsson GH, Sigurdsson JF, Asgeirsdottir BB, Sigfusdottir ID (2006) Custodial interrogation, false confession and individual differences. A national study among Icelandic youth. Personal Individ Differ 41:49-59

7. Gudjonsson GH, Sigurdsson JF, Sigfusdottir ID (2009) Interrogations and false confessions among adolescents in seven countries in Europe. What background and psychological factors best discriminate between false confessors and non-false confessors? Psychol Crime Law 15:711-728

8. Sigurdsson JF, Gudjonsson GH (2001) False confessions: The relative importance of psychological, criminological and substance abuse variables. Psychol Crime Law 7:275-289 
9. Young S, Moss D, Sedgwick O, Fridman M, Hodgkins P (2014) A meta-analysis of the prevalence of attention deficit hyperactivity disorder in incarcerated populations. Psychol Med. doi:10. 1017/S0033291714000762

10. Gudjonsson GH (2003) The psychology of interrogations and confessions. A handbook. Wiley, Chichester

11. Gudjonsson GH, Sigurdsson JF, Sigfusdottir ID, Young S (2012) False confessions to police and their relationship with conduct disorder, ADHD, and life adversity. Personal Individ Differ 52:696-701

12. Waschbusch DA (2002) A meta-analytic examination of comorbid hyperactive-impulsive-attention problems and conduct problems. Psychol Bull 128(1):118-150

13. Young S, Hopkin G, Perkins D, Farr C, Doidge A, Gudjonsson G (2013) A controlled trial of a cognitive skills program for personality-disordered offenders. J Atten Disord 17(7):598-607. doi: $10.1177 / 1087054711430333$

14. Thapar A, Harrington R, McGuffin P (2001) Examining the comorbidity of ADHD-related behaviours and conduct problems using a twin study design. Br J Psychiatry 179:224-229

15. Young S, Gudjonsson GH (2006) ADHD symptomatology and its relationship with emotional, social and delinquency problems. Psychol Crime Law 12:463-471

16. Lynam DR (1996) Early identification of chronic offenders: who is the fledgling psychopath? Psychol Bull 120(2):209-234

17. Gudjonsson GH, Sigurdsson JF, Sigfusdottir ID, Young S (2014) A national epidemiological study of offending and its relationship with ADHD symptoms and associated risk factors. J Atten Disord 18(1):3-13. doi:10.1177/1087054712437584

18. Barkley RA, Murphy KR, Fischer M (2008) ADHD in adults. What the science says. Guilford Press, London

19. Loeber R, Burke JD, Lahey BB, Winters A, Zera M (2000) Oppositional defiant and conduct disorder: a review of the past 10 years, part I. J Am Acad Child Adolesc Psychiatry 39(12):1468-1484. doi:10.1097/00004583-200012000-00007

20. Satterfield JH, Faller KJ, Crinella FM, Schell AM, Swanson JM, Homer LD (2007) A 30-year prospective follow-up study of hyperactive boys with conduct problems: adult criminality. J Am Acad Child Adolesc Psychiatry 46(5):601-610. doi:10.1097/chi. 0b013e318033ff59

21. Langley K, Fowler T, Ford T, Thapar AK, van den Bree M, Harold G, Owen MJ, O'Donovan MC, Thapar A (2010) Adolescent clinical outcomes for young people with attention-deficit hyperactivity disorder. Br J Psychiatry 196(3):235-240. doi:10. 1192/bjp.bp.109.066274

22. Gudjonsson GH, Sigurdsson JF, Bragason OO, Einarsson E, Valdimarsdottir EB (2004) Confessions and denials and the relationship with personality. Legal Criminol Psychol 9:121-133

23. Gudjonsson GH, Sigurdsson JF, Bragason OO, Newton AK, Einarsson E (2008) Interrogative suggestibility, compliance and false confessions among prisoners and their relationship with attention deficit hyperactivity disorder (ADHD) symptoms. Psychol Med 38(7):1037-1044. doi:10.1017/S0033291708002882

24. Gudjonsson GH, Sigurdsson JF, Einarsson E, Bragason OO, Newton AK (2010) Inattention, hyperactivity/impulsivity and antisocial personality disorder: which is the best predictor of false confessions? Personal Individ Differ 48:720-724

25. Medford S, Gudjonsson GH, Pearse J (2003) The efficacy of the appropiate adult safeguard during police interviewing. Legal Criminol Psychol 8:253-266

26. Barkley RA (1998) Attention deficit/hyperactivity disorder: a handbook for diagnosis and treatment, 2nd edn. Guilford Press, New York
27. Gudjonsson GH, Sigurdsson JF, Sigfusdottir ID, Asgeirsdottir BB (2008) False confessions and individual differences. The importance of victimisation among youth. Personal Individ Differ 45:801-805

28. Lewinsohn PM, Rohde P, Farrington DP (2000) The OADPCDS: a brief screener for adolescent conduct disorder. J Am Acad Child Adolesc Psychiatry 39(7):888-895. doi:10.1097/00004583200007000-00018

29. Sigfusdottir ID, Farkas G, Silver E (2004) The role of depressed mood and anger in the relationship between family conflict and delinquent behavior. J Youth Adolesc 33(6):509-522

30. Long JS, Freese J (2006) Regression models for categorical dependent variables using Stata, 2nd edn. Stata Press, College Station

31. StataCorp (2013) Stata statistical software: release 13. StataCorp LP, College Station

32. Gudjonsson GH (2010) Invited article. Psychological vulnerabilities during police interviews. Why are they important? Legal Criminol Psychol 15:161-175

33. Gonzalez RA, Velez-Pastrana MC, Ruiz Varcarcel JJ, Levin FR, Albizu-Garcia CE (2015) Childhood ADHD symptoms are associated with lifetime and current illicit substance-use disorders and in-site health risk behaviors in a representative sample of Latino prison inmates. J Atten Disord 19(4):301-312. doi:10. $1177 / 1087054712461690$

34. Young S, Wells J, Gudjonsson GH (2011) Predictors of offending among prisoners: the role of attention-deficit hyperactivity disorder and substance use. J Psychopharmacol 25(11):1524-1532. doi: $10.1177 / 0269881110370502$

35. Gonzalez RA, Gudjonsson GH, Wells J, Young S (2013) The role of emotional distress and ADHD on institutional behavioral disturbance and recidivism among offenders. J Atten Disord. doi:10. $1177 / 1087054713493322$

36. Gudjonsson GH (2014) Mental vulnerabilities and false confession. In: St-Yves M (ed) Investigative interviewing. The essentials. Carswell, Toronto, pp 191-222

37. Adler LD, Nierenberg AA (2010) Review of medication adherence in children and adults with ADHD. Postgrad Med 122(1):184-191. doi:10.3810/pgm.2010.01.2112

38. Thiruchelvam D, Charach A, Schachar RJ (2001) Moderators and mediators of long-term adherence to stimulant treatment in children with ADHD. J Am Acad Child Adolesc Psychiatry 40(8):922-928. doi:10.1097/00004583-200108000-00014

39. Lichtenstein P, Halldner L, Zetterqvist J, Sjolander A, Serlachius E, Fazel S, Langstrom N, Larsson H (2012) Medication for attention deficit-hyperactivity disorder and criminality. N Engl J Med 367(21):2006-2014. doi:10.1056/NEJMoa1203241

40. Arnold LE, Hodgkins P, Kahle J, Madhoo M, Kewley G (2015) Long-term outcomes of ADHD: academic achievement and performance. J Atten Disord. doi:10.1177/1087054714566076

41. Rees-Jones A, Gudjonsson G, Young S (2012) A multi-site controlled trial of a cognitive skills program for mentally disordered offenders. BMC psychiatry 12:44. doi:10.1186/1471-244X$12-44$

42. Young S, Goodwin EJ, Sedgwick O, Gudjonsson GH (2013) The effectiveness of police custody assessments in identifying suspects with intellectual disabilities and attention deficit hyperactivity disorder. BMC Med 11:248. doi:10.1186/1741-7015-11248 